\title{
PREVALENCIA DEL TRAUMA DENTOALVEOLAR EN PACIENTES ATENDIDOS EN EL SERVICIO DE URGENCIAS DE LA CLÍNICA CARLOS ARDIL.A LULLE DE FLORIDABLANCA - COLOMBIA, ENTRE 1998 Y 2002
}

${ }^{1}$ Juan Carlos León G., ${ }^{1}$ Elio Contreras M., ${ }^{2}$ Leonardo Pineda P., ${ }^{3}$ Carlos Fernando Galeano A.

${ }^{1}$ Residente de II año, posgrado de endodoncia, U. Santo Tomás, ${ }^{2}$ Odontólogo, U. Santo Tomás, Especialista en Endodoncia, C.O. C., Docente U. Santo Tomás, ${ }^{3}$ Odontólogo, U. Santo Tomás, Especialista en Cirugía Maxilofacial, U. El Bosque, Docente U. Santo Tomás

Autor responsable de correspondencia: Dr. Juan Carlos León G.

Correo electrónico: jcleongdentis@yahoo.com.mx

\begin{abstract}
RESUMEN
Objetivo: Determinar la prevalencia del trauma dentoalveolar de las Historias Clínicas, según la base de datos de la Clínica Carlos Ardila Lulle de Floridablanca - Colombia, en individuos atendidos en el servicio de urgencias mèdicas, durante los años 1998 a 2002.

Materiales y métodos: Con una metodología observacional de tipo descriptivo-retrospectivo, se analizaron 1235 historias clínicas de pacientes atendidos por emergencia, cuyo diagnóstico, según la clasificación internacional de enfermedades y causas de defunción (CIE), novena revisión de 1975, especificaron los códigos 873.6 ; 873.7 y 959.0, relacionados con el trauma dentoalveolar.

Resultados: Se encontró una prevalencia del $45.7 \%$ de trauma dentoalveolar con dientes afectados y una mayor frecuencia en hombres que en mujeres, así mismo los accidentes de tránsito aportaron el mayor número de eventos. La primera década de la vida presentó la mayor prevalencia de trauma de tejido blando, y, entre la segunda y tercera década la mayor prevalencia fue afectación dental.

Conclusiones: Es importante destacar que la mayor parte de los eventos de trauma dentoalveolar fueron diagnosticados por el médico general, y sólo en los casos severos se remitió a consulta con el cirujano maxilofacial. Así mismo, la actuación del endodoncista fue subvalorada. Cabe anotar que el 99.14\% de los eventos, recibió atención por algún tipo de aseguramiento, siendo la EPS la utilizada. [León JC, Contreras E, Pineda L, Galeano C. Prevalencia del trauma dentoalveolar en pacientes atendidos en el servicio de urgencias de la clínica Carlos Ardila Lulle de Floridablanca, Colombia, entre 1998 y 2002. Ustasalud Odontología 2004; 3: 32 - 40]
\end{abstract}

Palabras clave: Trauma dentoalveolar, Prevalencia, Servicio de urgencias

\section{PREVALENCE OF DENTAL TRAUMA IN PATIENTS ATTENDED BY EMERGENCY ROOM SERVICE OF CARLOS ARDILA LULLE HOSPITAL FROM FLORIDABLANCA - COLOMBIA FROM 1998 UNTIL 2002}

\begin{abstract}
Purpose: To determine the prevalence of dental trauma from the database of "Carlos Ardila Lulle" Hospital, from Floridablanca - Colombia, in people that attended emergency room service from 1998 until 2002.

Material and methods: An observational methodology (descriptive-retrospective), 1235 medical cards were analyzed in patients who came to emergency room service, by diagnostic classification of diseases and mortality causes (CIE), nineth edition from 1975; with 873.6, 873.7 and 959.0 codes, related to dental trauma.

Results: Medical cards from 1998 until 2002 were analyzed. A prevalence of 45.7\% of Dental Trauma was found with affected teeth. There was higher frequency among male than female. Car accidents are the highest number of events of dental trauma.

Conclusions: It is important to highlight that most of Dental Trauma events were diagnosed by the physician, and only in severe cases they were remitted to consultation with the maxilofacial surgeon. Validation of endodontics was not to value. $99.14 \%$ of the events were assisted by some insurance type, being the "EPS" the most used one.
\end{abstract}

Key words: Dental trauma, Prevalence, Emergency room. 


\section{INTRODUCCIÓN}

El trauma dentoalveolar es un evento que se puede presentar en cualquier edad, tiempo y lugar; por lo mismo es importante tener conocimiento de su etiología, teniendo en cuenta que depende de factores socioculturales y sociodemográficos del país en el que se realice el estudio.

Trauma se desprende de la palabra accidente, que de acuerdo a la Real Academia Española, se define como "suceso eventual o acción del que involuntariamente resulta daño para las personas o cosas". ${ }^{1}$ A su vez, el término traumatismo, se define como el "Conjunto de lesiones del revestimiento cutáneo que interesan un tejido, un órgano 0 un segmento de miembro, provocado accidentalmente por un agente externo", ${ }^{1,2}$ y el término dentoalveolar, se ajusta a la relación de los componentes adyacentes al diente/hueso alveolar del sistema estomatognático. ${ }^{3-5}$

Epidemiológicamente en Colombia los porcentajes del trauma dentoalveolar son un $16.7 \%$ de los pacientes que acuden a urgencias odontológicas, por fractura de dientes, como lo reporta el III Estudio Nacional de Salud Bucal, ${ }^{6,7}$ lo que hace que no alcance a tener la considerable relevancia respecto de su etiología, diagnóstico, manejo y control; pero este tema toma real importancia cuando los efectos se comparan con las consideraciones médico-legales, los costos de tratamientos ideales para la reconstrucción facial y/o estética, e inclusive la consideración laboral, con relación a incapacidades, permisos y otros aspectos en los que derive.

Países como México, Singapur, Japón, Australia, Turquia, Gran Bretaña, Arabia, Tanzania, Brasil, Italia, Suiza y Malasia, entre otros, han realizado estudios retrospectivos acerca de la incidencia del trauma dentoalveolar; se presenta en los diferentes grupos etáreos, con diferencias en el tipo de lesión, que se genera; no obstante los resultados cambian, según el género, entorno y la influencia cultural. $^{8-24}$

El traumatismo de los dientes afecta la pulpa dental directa 0 indirectamente; en consecuencia las consideraciones endodónticas son importantes al evaluar y tratar las lesiones dentales. ${ }^{25}$ Las lesiones de los dientes se presentan con mayor frecuencia en el grupo de 7 a 15 años de edad, con diversas actividades, como son los accidentes de tránsito, accidentes domésticos y deportes, los más reportados en la literatura. ${ }^{26}$
Es necesario tener en cuenta la clasificación de las lesiones traumáticas, con el objetivo de fomentar una mejor comunicación y divulgación de la información. ${ }^{27-29} \mathrm{El}$ sistema utilizado en este estudio, se basa en la clasificación de la Organización Mundial de la Salud (OMS) y la modificación realizada por Andreasen: ${ }^{30-32}$

- Fractura de esmalte

- Fractura de corona sin afectación pulpar

- Fractura de corona con afectación pulpar

- Fractura radicular

- Fractura corona raíz

- Luxación

- Avulsión

- Fractura del proceso alveolar

- Lesión de tejido blando

Los estudios clínicos retrospectivos revelaron diferentes distribuciones de la etiología dependiendo de si el material es recolectado en Hospitales, Clínicas Dentales Universitarias o Clínicas Públicas de Salud Dental.

Los accidentes de tráfico (ciclísticos) y accidentes domésticos son las dos principales etiologías de trauma dentoalveolar. ${ }^{33}$ Los deportes de contacto como el hockey sobre hielo, tienen mayor relación con injuria facial; ${ }^{34}$ pero deportes individuales, como la gimnasia, natación y equitación, favorecen el trauma dental. En Finlandia, el trauma dental más atendido en las Clínicas Odontológicas Públicas fue la afectación de tejidos duros, en contraste con la atención a pacientes en el Hospital Universitario de Dinamarca donde predominó la afectación periodontal. ${ }^{35}$

La mayoría de los autores, que han realizado estudios estadísticos (epidemiológicos) aceptan que es difícil hacer una comparación absoluta de las cifras obtenidas en un estudio, con las de otro, por los diversos tipos de subpoblación en los que se realizan los estudios. Sin embargo, muchas de las investigaciones de este tipo tienen en común varios hallazgos (tipo de lesión, actividad, edad); de este modo permiten conocer los tipos, la incidencia y la causa del trauma dentoalveolar. ${ }^{36,37}$

Los programas educacionales, acerca de la importancia del trauma dental, dirigidos a la prevención del mismo en beneficio de la atención inmediata, y conservación del diente avulsionado y fracturado son de gran ayuda para los pacientes..$^{38}$ Adicionalmente, proporcionan conocimiento al Odontólogo sobre el trauma, que es otro punto importante en la solución del problema. ${ }^{39,40}$ 
El objetivo del presente estudio observacional - descriptivo - retrospectivo es determinar la prevalencia del trauma dentoalveolar por año, género y edad, de acuerdo con las historias clínicas de los individuos atendidos en el servicio de urgencías de la Clínica Carlos Ardila Lulle, de Floridablanca, entre los años 1998 y 2002.

\section{MATERIALES Y MÉTODOS}

La población de estudio estuvo conformada por 1.235 historias clínicas de individuos, atendidos en el servicio de urgencias de la Clínica Carlos Ardila Lulle de Floridablanca, en el período comprendido entre 1998 y 2002, cuyo diagnóstico, según la Clasificación Internacional de Enfermedades y Causas de Defunción (CIE), en su novena revisión, de 1975, especificaron los códigos 873.6 - 873.7 y 959.0, relacionados con trauma dentoalveolar.

Se incluyeron las historias clínicas que registraban afectación de estructuras dentales y tejidos blandos de la cavidad oral, a los que se les aplicó el instrumento específico del estudio.

Se excluyeron aquellas historias clínicas que no tuvieran disponible la información requerida para el estudio 0 con otro tipo de trauma.

La muestra estuvo conformada por la totalidad de las historias clínicas que cumplieron con el criterio de inclusión definido y que hicieran parte del período de estudio, $n=116$. Las variables consideradas en el estudio se presentan en la Tabla 1.
Se aplicó el instrumento diseñado para este estudio. Posteriormente, se llevó a cabo la tabulación digitada en una hoja Excel, relacionando las diferentes variables descritas en el Tabla 1, para su posterior análisis.

Inicialmente se describieron las variables de estudio, según su naturaleza y distribución, aplicando medidas de tendencia central y de dispersión o tablas de frecuencia, y se estableció la prevalencia de trauma dentoalveolar general, y específico de diente.

Estas prevalencias fueron descritas por año para analizar la tendencia de los dos indicadores.

A continuación se definió, como variable dependiente, la lesión de trauma dentoalveolar (TDA) con diente afectado y sin diente afectado, contra la cual se evaluaron posibles asociaciones con las variables de estudio como género, edad, tipo de aseguramiento. Aplicando una prueba de $\mathrm{X}^{2}$ con un nivel de significancia $\alpha=0.05$.

Considerando el artículo 11 de la resolución 0080430 de 1993 del Ministerio de Salud de Colombia, este trabajo se consideró como una investigación sin riesgo.

\section{RESULTADOS}

La distribución por género mostró una frecuencia de 26.7\% para el género femenino y $73.3 \%$ para el masculino. La edad mostró un rango entre 1 y 70 años con un promedio de 18 años (Figura 1).

\begin{tabular}{cccc}
\hline Variable & Definición & Tipo & Nivel operativo \\
\hline Edad & Años cumplidos & Numérica & Años \\
Género & Definición de género & Nominal & Hombre - mujer \\
Aseguramiento & Tipo de aseguramiento & Nominal & Clasificación \\
Fecha & Especificar fecha del evento & Nominal & Día/mes/año \\
Diente afectado & Clasificación Internacional & Numérica & Según su ubicación \\
Actividad realizada & Factores predisponentes & Nominal & Nombre de la actividad \\
Diagnóstico & Según la OMS & Nominal & Nombre del diagnóstico \\
Profesional tratante & Formación académica & Nominal & Nombre de la profesión \\
Interconsulta odontológica & Negación - afirmación & Nominal & Si- No \\
Estado de conciencia & Afectación estado neurológico & Nominal & Consciente - inconsciente
\end{tabular}

Tabla 1. Descripción de las variables definidas para este estudio 


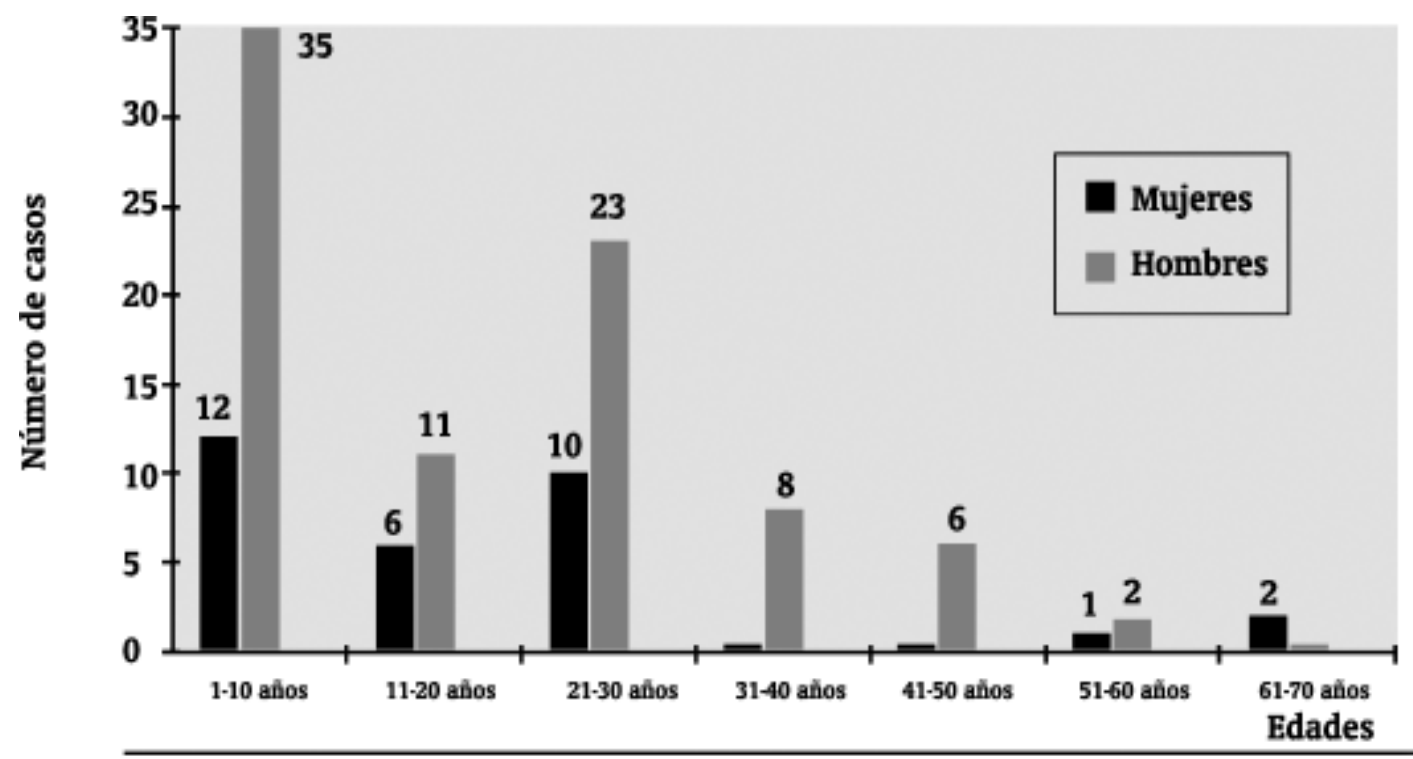

Figura 1. Trauma dentoalveolar en la población estudiada, según género y grupo de edad Fuente: Revisión Historias Clinicas Servicio de Urgencias. Clinica Carlos Ardila Lulle, 1998-2002

\begin{tabular}{ccccc}
\hline \multirow{2}{*}{$\begin{array}{c}\text { Grupos } \\
\text { de edad }\end{array}$} & Fem. & Mas. & \multicolumn{2}{c}{ Total } \\
\cline { 3 - 5 } & & & $n$ & $\%$ \\
\hline $1-10$ años & 5 & 14 & 23 & 43.4 \\
$11-20$ años & 5 & 9 & 14 & 26.4 \\
$21-30$ años & 5 & 5 & 10 & 18.9 \\
$31-40$ años & -- & 3 & 3 & 5.6 \\
$41-50$ años & -- & 2 & 2 & 3.8 \\
$51-60$ años & -- & 1 & 1 & 1.9 \\
61-70 años & -- & - & - & - \\
TOTAL & $\mathbf{1 9}$ & $\mathbf{3 4}$ & $\mathbf{5 3}$ & $\mathbf{1 0 0 \%}$ \\
\hline
\end{tabular}

Tabla 2. Distribución de casos con dientes afectados, según edad y género. Fuente: Revisión de Historias Clínicas Servicio de Urgencias. Clinica Carlos Ardila Lulle, 1998 - 2002.

La distribución de los casos de trauma dentoalveolar con dientes afectados, según el género, se presentó con mayor frecuencia en los hombres, con un total de 34 casos de los 53 registrados. Respecto de los grupos de edad, en la primera década se presenta el mayor número de casos con dientes afectados, con el 43.4\% (Tabla 2).

Respecto del registro de dientes afectados en los eventos de trauma dentoalveolar se presenta una prevalencia de un $45.7 \%$ sobre los 116 casos de trauma dentoalveolar

La prevalencia de trauma dentoalveolar con y sin afectación dental, según el año, se presenta en la Figura 2. En la descripción de la frecuencia por día de la semana se resalta una mayor frecuencia el día viernes con un $19.83 \%$, respecto de los demás días de la semana. En cuanto al mes, agosto representa el mayor porcentaje, con el 13.79\% $y$, con el menor porcentaje, enero con $2.59 \%$.

El tipo de aseguramiento se concentró en los items: Entidad Prestadora de Servicios de Salud (EPS) y Seguro Obligatorio contra Accidentes de Tránsito (SOAT), con un $43.9 \%$ y un $32.7 \%$, respectivamente.

Por el número de dientes afectados, a la vista de los eventos registrados por nomenclatura y número de dientes, de un total 30 casos, se presentó la mayor frecuencia con dos dientes afectados, en un 40\%, seguido por un solo diente afectado con el 33.3\%.

La frecuencia del trauma dentoalveolar, de acuerdo con la nomenclatura, muestra que los dientes incisivos centrales superiores 11 y 21 , con un $36.5 \%$ son los de mayor frecuencia. La sumatoria del total de dientes anteriores permanentes es de $53.9 \%$.

En el estudio se presentan los resultados del trauma dentoalveolar por diagnóstico; se registra un alto porcentaje de lesión de tejido blando, con un 53.4\%.

La distribución de los diferentes diagnósticos por edad y género, muestran mayor frecuencia del género masculino en lesión de tejidos blandos, con 52 casos (Tabla 3). La mayor actividad realizada durante el evento, se relaciona con accidentes de tránsito, con un 38.7\% 


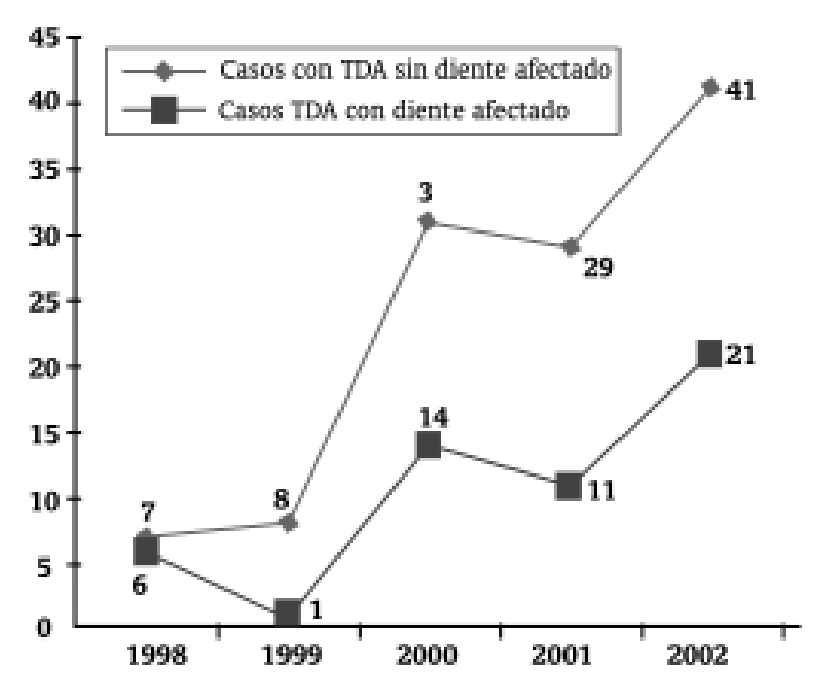

Figura 2. Prevalencia de trauma dentoalveolar con y sin afectación dental por año

Fuente: Revisión Historias Clinicas Servicio de Urgencias.

Clinica Carlos Ardila Lulle, 1998 - 2002

De los 53 casos que presentaron dientes afectados, el mayor porcentaje se registra en los accidentes de tránsito, con un $53.3 \%$.

En el 100\% de los casos, el paciente fue valorado inicialmente por el médico general del servicio de urgencias. La interconsulta con odontología (cirujano maxilofacial) sólo se realizó en un 18.9\% de los casos. Así mismo, el profesional que diagnosticó el trauma dentoalveolar se distribuyó de la siguiente manera: el $57.76 \%$, el médico general; el cirujano maxilofacial, 17.24\%; médico pediatra, $18.10 \%$, y otros el $6.9 \%$.

Análisis bivariado: La variable dependiente en el análisis bivariado tiene como base un $74.14 \%$ de los casos (86 individuos), representados en los diferentes grupos etáreos
(Tabla 4), que no registraron, según la historia clínica, compromiso de dientes en el trauma.

Los resultados muestran una asociación, estadísticamente significativa, para la edad, pues los grupos menores de 20 años, son los que muestran mayor frecuencia de lesiones dentales $p=0.025$. No se encontró una asociación significativa con género ni con tipo de aseguramiento ( $\mathrm{p}>0.05$ ).

De los tipos de actividades realizadas, se encontraron las mayores frecuencias por accidentes de tránsito, deportes y accidentes domésticos; sin embargo, los hallazgos no fueron significativos $(\mathrm{p}=0.115)$

En cuanto al profesional que diagnosticó el trauma dentoalveolar por diente afectado, se encontró la mayor frecuencia por cirujano maxilofacial, y médico general, asociación significativa $(\mathrm{p}<0.0001)$.

Igualmente, la interconsulta con odontología, en casos por diente afectado fue significativa ( $\mathrm{p}<0.001$ ), la cual se realizó en 17 casos con cirujano maxilofacial y un caso con odontología general.

No se encontraron asociaciones significativas por mes, año o día de la semana con el trauma dentoalveolar con afección dental.

\section{DISCUSIÓN}

El presente estudio se realizó con el objetivo de conocer la prevalencia del trauma dentoalveolar durante los años 1998 - 2002, por medio de un análisis epidemiológico, para determinar la relación entre los diferentes factores sociodemográficos y culturales, en la ocurrencia del trauma.

\begin{tabular}{|c|c|c|c|c|c|c|c|}
\hline \multirow[b]{2}{*}{ Diagnóstico } & \multicolumn{2}{|c|}{ Género } & \multicolumn{5}{|c|}{ Edad } \\
\hline & Masculino & Femenino & $\begin{array}{l}1 \cdot 10 \\
\text { años }\end{array}$ & $\begin{array}{l}11 \cdot 20 \\
\text { an̂os }\end{array}$ & $\begin{array}{l}21 \cdot 30 \\
\text { an̂os }\end{array}$ & $\begin{array}{l}31-40 \\
\text { años }\end{array}$ & $\begin{array}{l}\text { Más de } 40 \\
\text { años }\end{array}$ \\
\hline Fractura de esmalte & 1 & - & 1 & & & & \\
\hline Avulsión & 2 & 4 & 3 & 1 & 1 & 1 & - \\
\hline $\begin{array}{l}\text { Fractura proceso } \\
\text { alveolar }\end{array}$ & - & 1 & - & - & - & - & 1 \\
\hline Lesión tejido blando & 52 & 10 & 23 & 7 & 20 & 5 & 7 \\
\hline Otro & 11 & 9 & 6 & 5 & 7 & - & 2 \\
\hline Combinado & 19 & 7 & 14 & 4 & 5 & 2 & 1 \\
\hline TOTAL & 85 & 31 & 47 & 17 & 33 & 8 & 11 \\
\hline
\end{tabular}

Tabla 3. Distribución del diagnóstico del trauma dentoalveolar, por edad y género.

Fuente: Revisión Historias Clinicas Servicio de Urgencias. Clinica Carlos Ardila Lulle, 19982002 


\begin{tabular}{|c|c|c|c|}
\hline \multirow[b]{2}{*}{ Variable } & \multicolumn{2}{|c|}{ TDA } & \multirow[b]{2}{*}{ p } \\
\hline & $\begin{array}{c}\text { con afección } \\
\text { dental } \\
n=30\end{array}$ & $\begin{array}{c}\text { sin afección } \\
\text { dental } \\
n=86\end{array}$ & \\
\hline \multicolumn{4}{|l|}{ Edad (años) } \\
\hline $1-10$ & 12 & 35 & \multirow{4}{*}{0.025} \\
\hline $11-20$ & 10 & 7 & \\
\hline $21=30$ & 6 & 27 & \\
\hline$>30$ & 2 & 17 & \\
\hline Género masculino & 19 & 66 & 0.15 \\
\hline \multicolumn{4}{|l|}{$\begin{array}{l}\text { Tipo de } \\
\text { aseguramiento }\end{array}$} \\
\hline Ninguno & 0 & 1 & \multirow{6}{*}{0.31} \\
\hline ARS & 1 & 0 & \\
\hline Póliza estudiantil & 3 & 7 & \\
\hline EPS & 10 & 41 & \\
\hline SOAT & 13 & 25 & \\
\hline Otro & 3 & 12 & \\
\hline \multicolumn{4}{|l|}{ Actividad } \\
\hline Deporte & 4 & 8 & \multirow{9}{*}{0.115} \\
\hline Accidente de tránsito & 16 & 29 & \\
\hline Violencia intrafamiliar & 1 & 1 & \\
\hline Accidente doméstico & 4 & 16 & \\
\hline Accidente laboral & 0 & 4 & \\
\hline Accidente escolar & 2 & 1 & \\
\hline Riña & 1 & 4 & \\
\hline Sin especificar & 2 & 17 & \\
\hline Otro & 0 & 6 & \\
\hline \multicolumn{4}{|l|}{ Profesional } \\
\hline Médico general & 9 & 58 & \multirow{6}{*}{$<0.0001$} \\
\hline Cirujano maxilofacial & 16 & 4 & \\
\hline Odontólogo general & 1 & 1 & \\
\hline Médico, cirujano & 1 & 5 & \\
\hline general & 3 & 18 & \\
\hline Otro & & & \\
\hline Interconsulta OD & 18 & 4 & $<0.0001$ \\
\hline
\end{tabular}

Tabla 4. Análisis Bivariado.

Fuente: Revisión Historias Clinicas Servicio de Urgencias.

Clínica Carlos Ardila Lulle. 1998 - 2002

La población se sacó de los registros de ingreso en el servicio de urgencias, con base en la Clasificación Internacional de Enfermedades (CIE), Novena Edición, y los códigos 873.6; 873.7 y 950.9, vigentes hasta el año 2002. Se relacionan con traumatismos ó lesiones de la cavidad oral (cara y cuello), entre los cuales se especifica fractura de dientes. ${ }^{42}$

En una población de 1.235 historias clínicas al diligenciar el instrumento, se obtuvo una muestra de 116 casos, con manifestaciones clínicas de trauma dentoalveolar. Cuando el trauma dentoalveolar es diagnosticado por el médico general tiene poca aplicabilidad la clasificación de la OMS, elegida para realizar el presente estudio. ${ }^{31}$ Los diagnósticos se presentaron en una forma descriptiva como, por ejemplo, "diente flojo", "fractura de diente", "fractura de molares", "diente desplazado", que no son específicos para dicha clasificación, aunque aportan información sobre el compromiso de dientes en el evento del trauma dentoalveolar.

Los casos de trauma dentoalveolar con dientes afectados, se tomaron según la clasificación de la lesión, donde se especifica el compromiso dental y los registros como diagnóstico "otro", con dientes comprometidos, dando una muestra de eventos de trauma con afectación dental de 53 casos, que representa un $45.68 \%$ del total de eventos de trauma con real afectación dental. Para calcular la frecuencia de diente más afectado y el número de dientes comprometidos en cada evento, se tuvieron en cuenta sólo las historias clínicas, que aportaban esta información, con un total de 30 casos, con su nomenclatura y cantidad de dientes afectados.

La distribución por género mostró una mayor frecuencia en el género masculino con un $73.3 \%$, y un $23.7 \%$ para el género femenino, con una relación de 2.7, siendo semejante a la observada en otros estudios, que examinaron pacientes atendidos en un servicio de urgencias, si bien difiere de los estudios realizados en la población escolar, en los diferentes centros educativos públicos o privados; en estos la relación es baja, hasta de 1:1. La relación entre los géneros en la primera década es de 2.9, teniendo un cambio en la segunda y tercera década de 1.8 y 2.3 , respectivamente. Esto podría explicarse por cuanto las mujeres tienden a una mayor participación en los eventos de accidentes de tránsito, llegando a tener en la tercera década una relación 1:1 respecto de los hombres.

Respecto del tiempo de ocurrencia hay que anotar que la prevalencia en el día de la semana no tiene relevancia estadística; la más alta prevalencia se presentó el día viernes, con un $19.83 \%$ seguido por el día lunes, con un 17.24\%. Al agrupar los tres últimos días (viernes, sábado y domingo) se encontró que casi la mitad de los eventos, $48.28 \%$, ocurren en estos días; sin embargo, no fueron significativos $(\mathrm{p}=0.47)$.

Respecto del mes, en el presente estudio no existe una asociación significativa como en otros estudios en que aumenta en los meses de invierno y verano, cuando los escolares se encuentran en época de vacaciones. Es importante anotar que en nuestra región, por su ubicación ecuatorial, no existen las estaciones y, por lo tanto, no pueden compararse los resultados. Sin embargo, la tendencia claramente muestra dos ciclos durante el año, que inician con cifras 
bajas en enero y junio, y se incrementan paulatinamente en mayo y noviembre. Lo cual coincide con el período escolar, pero no es significativo, puesto que el accidente escolar tiene una baja frecuencia.

La población se distribuyó por años, con el mayor número de los casos en el 2001 con 363, muy similar a los años 1999, 2000 y 2002, y el año 1998 con 44 casos, que muy probablemente se debió a un inadecuado registro, por el comportamiento de los años subsiguientes. La distribución del trauma dentoalveolar por año, indica una frecuencia del 9.4\% con respecto a la población estudiada.

En el año 1998 se observó mayor frecuencia, con un 15.4\% y en el 2000 con $14.7 \%$ de total de las historias revisadas; son muy similares sin diferencias significativas. Al evaluar los casos con dientes afectados el mayor número se presentó en el año 2002, manteniendo una tendencia proporcional respecto de la ocurrencia del trauma dentoalveolar, en los demás años.

El profesional que describe el diagnóstico del trauma dentoalveolar, más frecuentemente, es el médico general, con un 57.76\%, que se relaciona con el sitio donde se atendió la emergencia; ellos son los que tienen entrenamiento para la atención de urgencias. Pero teniendo en cuenta que los pacientes ingresaron en un estado consciente en un $99.14 \%$ de los casos presentados, sólo recibieron valoración por consulta odontológica (cirujano maxilofacial) el 17.24\% (20 casos), siendo el único especialista tenido en cuenta, subutilizando la disponibilidad del endodoncista, ${ }^{39}$ que está entrenado y capacitado para diagnosticar y tratar el trauma dentoalveolar, minimizando las complicaciones y las secuelas.

También cabe destacar la participación del médico pediatra en 21 casos, asociado con la presencia de trauma dentoalveolar en niños; estos casos, se consideran deberían ser tratados por el servicio odontológico, y en especial por el odontopediatra.

Al analizar la descripción del diagnóstico de los casos atendidos, en el servicio de urgencias de la Clínica Carlos Ardila Lulle, un alto porcentaje de afectación se presentó en los tejidos blandos, con un 53.4\%, cuando fue descrito como único diagnóstico, al presentarse el doble diagnóstico, en asocio con diente afectado, este porcentaje ascendió al 69.09\%, presentándose una mayor frecuencia en el género masculino y en la primera década con un $64.15 \%$, que se correlaciona con estudios retrospectivos de otros autores, como el realizado por Llarena del Rosario y colaboradores, en México, en el que reportan trauma de tejidos blandos en la población infantil en un $62.3 \%$; $^{9}$ y también con otros estudios realizados en subpoblación infantil, como el registro de Pérez y colaboradores con un $58 \%{ }^{43}$

En edades entre la segunda y tercera década, en el presente estudio, es la injuria de los tejidos blandos la de mayor frecuencia; en contra de los estudios que reportan como principal injuria la fractura del esmalte y dentina.

La mayoría de los eventos son casos severos; en ellos se involucran tejidos blandos y múltiples dientes, como los encontrados en otros estudios realizados en servicios de emergencia. ${ }^{447}$ Los dientes más frecuentemente afectados son los incisivos centrales superiores (11 y 21), con un porcentaje de $36.5 \%$ de 63 dientes afectados, debido a su ubicación anatómica. Estos resultados se correlacionan con todos los estudios reportados a nivel mundial, y con el estudio de Hasbon y colaboradores, realizado en el Instituto de Medicina Legal y Ciencias Forenses de Bucaramanga, entre los años de 1994 - 1995, que reporta un $59.3 \%$ de frecuencia para los incisivos centrales superiores, sobre 165 dientes afectados. ${ }^{48}$

Estudios retrospectivos realizados por Andreasen, muestran que mientras en Dinamarca los eventos de trauma dentoalveolar se dan en un alto porcentaje en actividades deportivas, ${ }^{26}$ en este estudio predominan los accidentes de tránsito y domésticos, siendo los más frecuentes los accidentes de moto, asociados con consumo de bebidas alcohólicas, concentrándose el mayor número de casos entre los 21 y 30 años, con un 38.6\% de los casos, con una relación 1:1 entre hombres y mujeres. Respecto del accidente doméstico, se presenta en la primera década con un 83.3\%, considerando a los accidentes infantiles como domésticos, debido a que el menor está bajo la responsabilidad de sus padres o de un adulto.

El 99.14\% de los casos fueron atendidos por algún tipo de seguro del Sistema General de Seguridad Social. Es interesante observar que siendo el accidente de tránsito el de mayor porcentaje de trauma dentoalveolar, al correlacionarlo con el tipo de seguro con el cual fue atendido el paciente, son las EPS las que se utilizan con mayor frecuencia, 42.9\%; se pensaría que debería ser el SOAT, 
pero se concluye que se aumenta en las EPS porque aquí se asocian otros eventos como los deportivos, accidentes laborales y accidentes domésticos, lo que hace que se eleven los casos atendidos.

Al realizarse el estudio en un servicio de urgencias médicas, el diagnóstico del trauma dentoalveolar, se basa en la descripción del evento. El presente estudio tiene como referencia la clasificación de la OMS, que puntualiza los diagnósticos del trauma dentoalveolar. Por lo tanto, pierde relevancia en la información puntual del diagnóstico del trauma dentoalveolar. Así mismo, se limitó sólo a un servicio de urgencia, del área de influencia.

Adicionalmente, el estudio puede ser aplicado en otras instituciones, para el manejo epidemiológico del trauma dentoalveolar.

Se concluye que:

- La prevalencia del trauma dentoalveolar, con diente afectado, es de un $45.7 \%$, de los 116 casos de trauma dentoalveolar.

- La etiología del trauma dentoalveolar, estudiada en la población que acude a los servicios de urgencias de la Clínica Carlos Ardila Lulle, muestra más frecuencia en la lesión de tejido blando, con un 53.4\% y con mayor frecuencia en la primera década de la vida.

- El grupo de menores de 20 años presenta la mayor frecuencia de lesiones dentales; en su mayoría están asociadas con lesiones del tejido blando; los dientes centrales superiores son los más afectados.

- En cuanto a la actividad, la mayor frecuencia es por accidentes de tránsito; la mayoría de ellos en motos, asociado con el consumo de bebidas alcohólicas; en segundo lugar, los accidentes domésticos, en su mayoría en menores de 10 años.

- El 99.14\% de los casos de trauma dentoalveolar fueron atendidos por algún tipo de aseguramiento del Sistema General de Seguridad Social en Salud, siendo el más utilizado el Régimen Contributivo (EPS).

- En el servicio de urgencias de la Clínica Carlos Ardila Lulle, la interconsulta odontológica fue del 18.9\% y sólo con el cirujano maxilofacial.
- Así mismo, se subutiliza la acción del Endodoncista, que tiene el entrenamiento adecuado para el manejo del trauma dentoalveolar.

\section{BIBLIOGRAFÍA}

1. Enciclopedia Larousse. Disco compacto versión 2003.

2. Martines F, y colaboradores. Salud Pública. España: Mc. Graw Hill Hispanoamericana, 1998.

3. Cohen S, Burns RC. Vías de la pulpa. Capítulo 15. 7 ed., Madrid, España: Harcourt, 1999.

4. Ingle JI, Bakland LK. Endodontics. 4 ed., Baltimore: William \& Wilkins, 1994.

5. Weine F. Endodontic therapy. Capítulo 10. 4 ed., Estados Unidos: Mosby, 1989.

6. Universidad Industrial de Santander. Módulo 1. Biometría. Unidad de Epidemiología. Lectura 7. Bucaramanga: UIS, Postgrado de Administración de Servicios de Salud, 1998. p. 129

7. Ministerio de Salud, Colombia. Tercer Estudio Nacional de Salud Bucal, ENSAB III. Bogotá: Ministerio de Salud. República de Colombia, 1998. Tomo VII.

8. Sánchez AV, García-Godoy F. Traumatic dental injuries in 3 to 13 year old boys in Monterrey, México. Endod Dent Traumatol 1990; 6: 63 - 65.

9. Llarena del Rosario ME. Acosta VM, García-Godoy F. Traumatic injuries to primary teeth in México city children. Endod Dent Traumatol 1992; 8: 213 - 214.

10. Sae - Lim V, Tan HH, Yuen KW. Traumatic dental injuries at the Accidentand Emergency Department of Singapore General Hospital. Endod Dent Traumatol 1995; 11: 32 - 36.

11. Tsukiboshi M. Treatment planning for traumatized teeth. Quintessence Publishing Co. Inc. Japón: 2000.

12. Uji T, Teramoto T. Ocurrence of traumatic injuries in the oromaxillary region of children in a japanese prefecture. Endod Dental Traumatol 1988; 4: 63 - 69.

13. Bastone EB, Freer TJ, McNamara JR. Epidemiology of dental trauma: A review of literature. Australian Dent J 2000; 45: 2 - 9.

14. Caliskan MK, Turkun M. Clinical investigation of traumatic injuries of permanent incisors in Izmir, Turkey. Endod Dent Traumatol 1995; 11: $210-213$.

15. Altay N, Güngor HC. A retrospective study of dento-alveolar injuries of children in Ankara, Turkey. Dent Traumatol 2001; 17: 201 - 204. 
16. Hamilton FA, Hill FJ, Hollway PJ. An investigation of dento-alveolar trauma and its treatment in an adolescent population. Part 1: The prevalence and incidence of injuries and the extent and adequacy of treatment received. Br Dent J 1997; 182: 91 - 95.

17. Al M, Murria JJ, Maquire A. The prevalence of dental trauma in 56 and 12-14 years old boys in Riyadh, Saudi Arabia. Dent Traumatol 2001; 17: 153 - 158.

18. Kahabuka FK, Plaschaert A, Hof MA. Prevalence of teeth with untreated dental trauma among nursery and primary school pupils in Dar es Salaam, Tanzania. Dent Traumatol 2001; 17: 109 - 113.

19. Cortés M, Marcenes W, Sheiham A. Prevalence and correlates of traumatic injuries to the permanent teeth of school-children aged 9 - 14 years in Belo Horizontal, Brazil. Dent Traumatol 2001; 17: 22 - 26.

20. Cunha RF, Pugliesi DMC, Vieira AEM. Oral trauma in Brazilian patients aged $0-3$ years. Dent Traumatol 2001; 17: 210 - 212.

21. Nicolau B, Marcenes W, Sheiham A. Prevalence, causes and correlates of traumatic dental injuries among 13 year olds in Brazil. Dent Traumatol 2001; 17: 213 - 217.

22. Zerman N, Cavalleri G. Traumatic injuries to permanent incisors. Endod Dent Traumatol 1994; 9: 61 - 64.

23. Schatz JP, Joho JP. A retrospective study of dento-alveolar injuries. Geneve, Suiza. Endod Dent Traumatol 1994; 10; 11 - 14.

24. Nik - Hussein NN. Traumatic injuries to anterior teeth among school children in Malaysia. Dent Traumatol 2001; 17: 149 - 152.

25. Krasner A, Rankow HJ. New philosophy for the treatment of avulsed teeth. Oral Surg Oral Med Oral Pathol Oral Radiol 1995; 79: 616 - 623.

26. Andreasen JP, Andreasen FM. Dental Traumatology: Quo Vadis. Endod Dent Traumatol 1990; 6: 78.

27. García-Godoy F. A classification for traumatic injuries to primary and permanet teeth. J Pedod 1981; 5: 295 - 297.

28. Ellis RG. The classification and treatment of injuries to the teeth of children. 5 ed., Chicago: Year Book Medical Publishers, 1970: 56 - 199.

29. Sweet CA. A classification and treatment for traumatized anterior teeth. J Dent Child 1955; 22: 144 -149.

30. World Health Organization. Application of the International Classification of Diseases to Dentistry and Stomatology (IDC DA). 2 ed., Geneva, Suiza: WHO Organization, 1978: 88 - 89.

31. World Heart Organization. Application of the International Classification of Diseases to Dentristy and Stomatology (IDC - DA). 3 ed., Geneva, Suiza, 1993.

32. Andreasen JP, Andreasen FM. Textbook and color atlas of traumatic injuries to the teeth. 3 ed., San Luois, Estados Unidos: Mosby, 1994.
33. Alonge OK, Narendran S, Williamson DD. Prevalence of fractured incisal teeth among children in Harris County, Texas. Dent Traumatol 2001; 17: 218 - 221.

34. Sane J, Ylipaavalniemi P. Dental trauma in contact team sports. Endod Dent Traumatol 1988; 4: 164 -169.

35. Oikarinen $\mathrm{K}$, Kassila O. Causes and types of traumatic tooth injuries treated in a public dental health clinic. Endod Dent Traumatol 1987; 3: 172 - 177

36. Luz JGC, Di Mase F. Incidence of dentoalveolar injuries in hospital emergency room patients. Endod Dent Traumatol 1994; 10: $188-190$.

37. Caldas AF, Burgos M. A retrospective study of traumatic dental injuries in a Brazilian dental trauma clinic. Dent Traumatol 2001; 17: $250-253$.

38. Westerman B, Stringfellow PM, Eccleston JA. EVA mouthguards: how thick should they be? Dent Traumatol 2002; 18: 24 - 27.

39. Barnett $F$. The role of endodontics in the treatment of luxated permanent teeth. Dent Traumatol 2002; 18: $47-56$.

40. Meir R, Keating J, Croll TP. Traumatic impaction of a maxillary primary incisor into the nasal cavity. J Pedod 1985; 9: 338 - 343.

41. Gábris K., Tarján I, Rózsa N. Dental trauma in children presenting for treatment at the Departament of Dentistry for Children and Orthodontics. Budapest, 1985-1999. Dent Traumatol 2001; $17: 103-108$

42. Clasificación Internacional de Enfermedades y Causas de defunción. (CIE). 9na Edición, 1975,

43. Perez R, Berkowitx R, Mcllveen I Forrester 0. Dental trauma in children: a survey. Endod Dent Traumatol 1991; 7: $212-213$.

44. Majorana A, Pasini S, Bardellini E, Keller E. Clinical and epidemiological study of traumatic root fractures. Dent Traumatol 2002; 18: 77 - 80.

45. O'Neil DW. Oral trauma in children: A hospital survey. Oral Surg Oral Med Oral Pathol 1989; 68: 691.

46. Rocha MJC, Cardoso, M. Traumatized permanent teeth in Brazilian children assisted at the Federal University of Santa Catarina, Brasil. Dent Traumatol 2001; 17: $245-249$.

47. Galea H. An investigation of dental injuries treated in an acute care general hospital. J Am Dent Assoc 1984; 109: 434 - 438.

48. Hasbon JC, Pineda VM. Relación entre el tratamiento del trauma orofacial y su incidencia en el dictamen médico legal. Bucaramanga, 1995. Trabajo de Grado (Odontólogo). Universidad Santo Tomás. Facultad de Odontología. 